\title{
The Roles of Wind Shear and Thermal Stratification in Past and Projected Changes of Atlantic Tropical Cyclone Activity
}

\author{
Stephen T. Garner, IsaAc M. Held, Thomas Knutson, and Joseph Sirutis \\ Geophysical Fluid Dynamics Laboratory, Princeton, New Jersey
}

(Manuscript received 13 November 2008, in final form 23 March 2009)

\begin{abstract}
Atlantic tropical cyclone activity has trended upward in recent decades. The increase coincides with favorable changes in local sea surface temperature and other environmental indices, principally associated with vertical shear and the thermodynamic profile. The relative importance of these environmental factors has not been firmly established. A recent study using a high-resolution dynamical downscaling model has captured both the trend and interannual variations in Atlantic storm frequency with considerable fidelity. In the present work, this downscaling framework is used to assess the importance of the large-scale thermodynamic environment relative to other factors influencing Atlantic tropical storms.

Separate assessments are done for the recent multidecadal trend (1980-2006) and a model-projected global warming environment for the late 21 st century. For the multidecadal trend, changes in the seasonal-mean thermodynamic environment (sea surface temperature and atmospheric temperature profile at fixed relative humidity) account for more than half of the observed increase in tropical cyclone frequency, with other seasonal-mean changes (including vertical shear) having a somewhat smaller combined effect. In contrast, the model's projected reduction in Atlantic tropical cyclone activity in the warm climate scenario appears to be driven mostly by increased seasonal-mean vertical shear in the western Atlantic and Caribbean rather than by changes in the SST and thermodynamic profile.
\end{abstract}

\section{Introduction}

Tropical cyclone activity shows its greatest variability in the North Atlantic region, where hurricane counts can vary interannually from just a few (e.g., one in 1982) to more than a dozen (e.g., fifteen in 2005). Since the 1970s, several measures of Atlantic tropical cyclone activity have trended upward, with a fairly sharp increase occurring in the mid-1990s. The number of tropical storms during the 3-month season from August to October, as modeled by the linear trend, increased from 6 to 12 between 1980 and 2005 (e.g., Knutson et al. 2007, hereafter K07). The accumulated cyclone energy (ACE) has been above average every year since 1994 except the El Niño years of 1997 and 2002 (e.g., Bell et al. 2006) and the 2007 season. Emanuel $(2005,2007)$ reports a substantial increase in the power dissipation index in the Atlantic since 1950.

Corresponding author address: Stephen T. Garner, Princeton University, Forrestal Campus, 201 Forrestal Road, Princeton, NJ 08540-6649.

E-mail: steve.garner@noaa.gov
Correlations with indices of large-scale conditions have been used to try to understand the interannual and longer-term variability of tropical storm activity (Gray et al. 1994). Examples of these from recent studies include sea surface temperature and hurricane potential intensity (PI; Emanuel 2007), local SST as a deviation from the tropical mean (Swanson 2008), moist static stability (Tang and Neelin 2004), vertical shear (Goldenberg and Shapiro 1996), low-level winds (Saunders and Lea 2008), and an index combining hurricane potential intensity, shear, vorticity, and midlevel relative humidity (Nolan et al. 2007).

Vertical shear has an adverse effect on tropical cyclone formation (Gray 1968; Goldenberg et al. 2001; Camargo et al. 2007) and on storm strength and longevity (Gray 1968; Hebert 1978; Merrill 1988; DeMaria 1996). In the tropical Atlantic, vertical shear is determined by gradients of SST both locally within the basin and remotely from the Indo-Pacific. High static stability is also an adverse influence on tropical cyclones (e.g., DeMaria et al. 2001; Shen et al. 2000) because it both suppresses deep convection during cyclogenesis and 
reduces the potential intensity (Emanuel 1986; Holland 1997) of organized storms. This stability is mainly determined by the contrast between SST and uppertropospheric air temperature. Upper-tropospheric temperature, in turn, covaries with tropical-mean sea surface temperature, helping to explain why Atlantic hurricane activity is strongly correlated with the deviation of Atlantic SST from the tropical mean (Swanson 2008).

Although statistical empirical models have been a mainstay in seasonal forecasting, dynamical models now show promise of generating significant skill at reproducing and predicting tropical storm counts. Vitart et al. (2007) describe a dynamical framework that considerably outperforms two well-known statistical forecast systems during the period 1993-2006, matching the observed hurricane counts with a correlation coefficient of 0.81 .

A downscaling procedure using observed large-scale atmospheric conditions and SST can be expected to reproduce past tropical storm frequencies more accurately than a coupled model. Adopting this approach, K07 achieved a 0.84 correlation between observed and simulated August-to-October hurricane counts in the North Atlantic between 1980 and 2005, based on two realizations. The study used a high-resolution, nonhydrostatic atmospheric model forced at the boundaries by reanalysis fields and in the interior by relaxation toward the large-scale component of the reanalysis (defined as zonal and meridional wavenumbers $0-2$ in their Atlantic domain). This framework evidently captures many of the precursors and dynamical processes important to cyclogenesis.

Because it allows the model environment to be altered, the "perfect large-scale conditions" platform of K07 is ideal for sorting out the relative importance of different sources of variability. As the dynamical fidelity of such models improves, one should be able to determine sensitivities to slowly varying environmental conditions with greater reliability than through use of multiple regression analysis of observations.

Because the framework in $\mathrm{K} 07$ is based on a physical model, its use in climate change applications may be more justified than is the case for statistical models trained on present-day climate, once its performance for the past is deemed adequate. In such a climate change application, Knutson et al. (2008, hereafter K08) find that storm counts in the North Atlantic decrease by the end of the century because of global warming. This result is no doubt sensitive to details of the presumed climate change pattern. A particularly valuable use of downscaling would be to attribute the projected changes in hurricane frequency to specific components of the projected climate change. Reducing uncertainty in projected tropical upper-tropospheric temperatures or
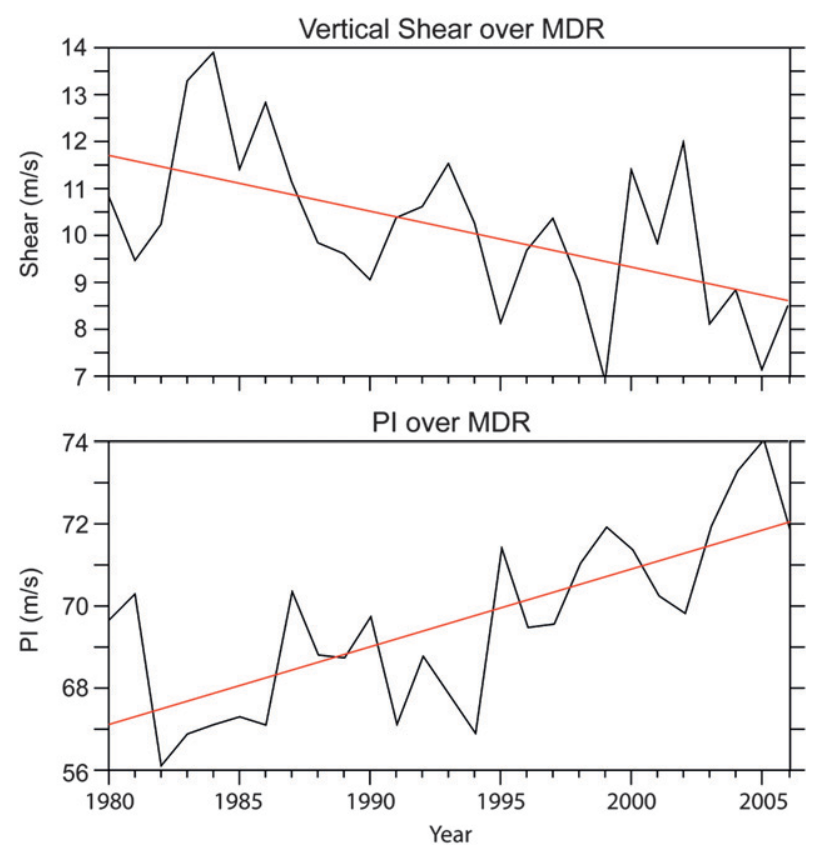

FIG. 1. Time series of MDR-averaged (top) vertical shear and (bottom) PI (both in $\mathrm{m} \mathrm{s}^{-1}$ ) evaluated from ASO averages of the NCEP reanalysis fields. The linear trends are also shown. They are $-0.12 \mathrm{~m} \mathrm{~s}^{-1} \mathrm{yr}^{-1}$ for the shear and $+0.19 \mathrm{~m} \mathrm{~s}^{-1} \mathrm{yr}^{-1}$ for the PI. The difference of vertical shear between the highest and lowest terciles of seasons based on tropical storm count is $-1.2 \mathrm{~m} \mathrm{~s}^{-1}$, while the difference of PI averages is $+2.8 \mathrm{~m} \mathrm{~s}^{-1}$.

wind shear, for example, becomes more urgent if these factors are shown to have a crucial influence on hurricane frequency.

In this study we use the framework documented in K07 for two sensitivity experiments. Through these, we attempt to separate the roles of vertical shear and thermodynamic stability in the recent storm frequency trend as well as in the projected storm suppression due to 21stcentury climate change.

Year-to-year variations of vertical shear and potential intensity from 1980 to 2006 are shown in Fig. 1. The shear is the magnitude of the vector wind difference between 850 and $200 \mathrm{hPa}$, while the potential intensity is based on the algorithm of Bister and Emanuel (2002). Specifically, the PI assumes irreversible ascent and includes dissipative heating. These choices are consistent with the way the numerical model was set up. Both the shear and PI are evaluated from seasonal-mean reanalysis fields and then averaged over the North Atlantic main development region (MDR). For the purposes of this study, we define the MDR as the region from $10^{\circ}$ to $23^{\circ} \mathrm{N}$ latitude and from $20^{\circ}$ to $80^{\circ} \mathrm{W}$ longitude. The data are from the NCEP-1 Reanalysis (Kalnay et al. 1996), which we have also used for driving the numerical experiments. 
The trend during the 1980-2006 period is toward less shear (down 30\%) and more potential intensity (up 7\%). Both trends are qualitatively consistent with the observed increases in Atlantic storm activity. The PI increase is mainly driven by the increasing local SST combined with minimal changes in upper-tropospheric temperature over the tropical Atlantic in the reanalysis (Vecchi and Soden 2007b). Vitart and Anderson (2001), Goldenberg et al. (2001), and Hoyos (2006) also noted increasingly favorable shear, SST, and/or moist stability during this period in the tropical North Atlantic.

Because of changes in observing instruments, there is uncertainty about the validity of the trends in NCEP tropical temperature profiles (Karl et al. 2006; Santer et al. 2005). The regional model used in our study, as described in K07, captures the sign and rough magnitude of the recent multidecadal trends in Atlantic tropical cyclone activity. However, to the extent that the model trend is caused by the trend in the thermodynamic profile, some of the model storm increases could be spurious. Global climate models project continued sea surface warming due to greenhouse gas forcing but do not foresee a large increase in PI over the tropics as a whole or over the tropical Atlantic in particular (Vecchi and Soden 2007b).

Vertical shear and PI are quasi-independent indices that have commonly been used to interpret and predict storm frequency. We use them here as a convenient way to characterize the imposed environments, even though the latter have many more degrees of freedom. For Fig. 1, we computed the indices from seasonal-mean conditions because we are interested in whether observed variability of tropical cyclone activity can be reproduced using only seasonal means of the influencing fields. Performing the time averaging after evaluating the PI has a small impact, in any case. There is a larger impact on the vertical shear, but the additional contribution to this shear from the subseasonal variance of the wind (not shown) is concentrated mostly outside the deep tropics and exhibits less interannual variability.

\section{The model and methodology}

The numerical model (Pauluis and Garner 2006) and domain used here are the same as in K07. The model's dynamical core is compressible and nonhydrostatic, with a terrain-following vertical coordinate. The domain covers the tropical and subtropical Atlantic, Gulf of Mexico, and parts of western Africa. The grid spacing is $1 / 6^{\circ}$ and there are 45 unevenly spaced levels. Sea surface temperatures are specified from the NCEP reanalysis, and a simple land model (Milly and Shmakin 2002) predicting soil temperature and moisture is used at land points. All precipitation occurs on resolved scales; there is no cumulus closure scheme. The boundary layer turbulence, microphysics, and radiative transfer schemes are described in K07.

Our implementation of the "perfect large-scale conditions" method is also exactly as in K07. Within a graduated $5^{\circ}$-wide band around the perimeter of the domain, the velocity, temperature, and humidity are nudged toward the time-interpolated 6-hourly reanalysis on a fast time scale $(2 \mathrm{~h})$. Across the entire domain, zonal and meridional wavenumbers 0,1 , and 2 of the model domain are nudged toward the reanalysis on a slower time scale $(36 \mathrm{~h})$. The nudging keeps the model's solution similar to the NCEP reanalysis on the large scale, but the model remains relatively unconstrained in generating smaller-scale disturbances within that environment.

Modeling studies to determine hurricane sensitivity to the large-scale environment have generally used idealized perturbations of the static stability or shear (e.g., Nolan et al. 2007; Shen et al. 2000). In contrast, we define the perturbations using observed or projected long-term trends. We limit our perturbations to 3-month-mean fields-specifically, averages over August, September, and October (ASO). The success of seasonal hurricane forecasts leads us to expect a substantial response to the seasonal-mean anomalies. In the following, for convenience, we refer to the departure from the seasonal mean in the input fields, both in the model interior and at the boundary, as the "weather" input. The present framework allows us to compare the importance of the seasonal mean to that of the weather in driving the model's tropical storm variability.

Attempts to separately perturb different components of the environment are complicated by the dynamical relationship between mass and wind fields. Perturbing the wind generally requires changing the static stability (if there is curvature in the wind profile, the static stability must vary in the horizontal). Inversely, one can alter the static stability without changing the wind field only if the alteration is horizontally uniform.

We therefore settle on two different types of perturbation in this initial study: 1) changes to all seasonalmean fields at once (all-fields) and 2) changes to the horizontally averaged temperature profile and SST, holding the relative humidity fixed ( $T$-only). The second perturbation leaves the wind unchanged. The anomaly in the temperature profile and SST, which we apply uniformly across the full domain, is taken to be the horizontal average over the MDR from the National Centers for Environmental Prediction (NCEP) reanalysis. This single-column perturbation does not capture horizontal variations of moist stability. To the extent that the response is linear, we can infer the impact of all effects not 

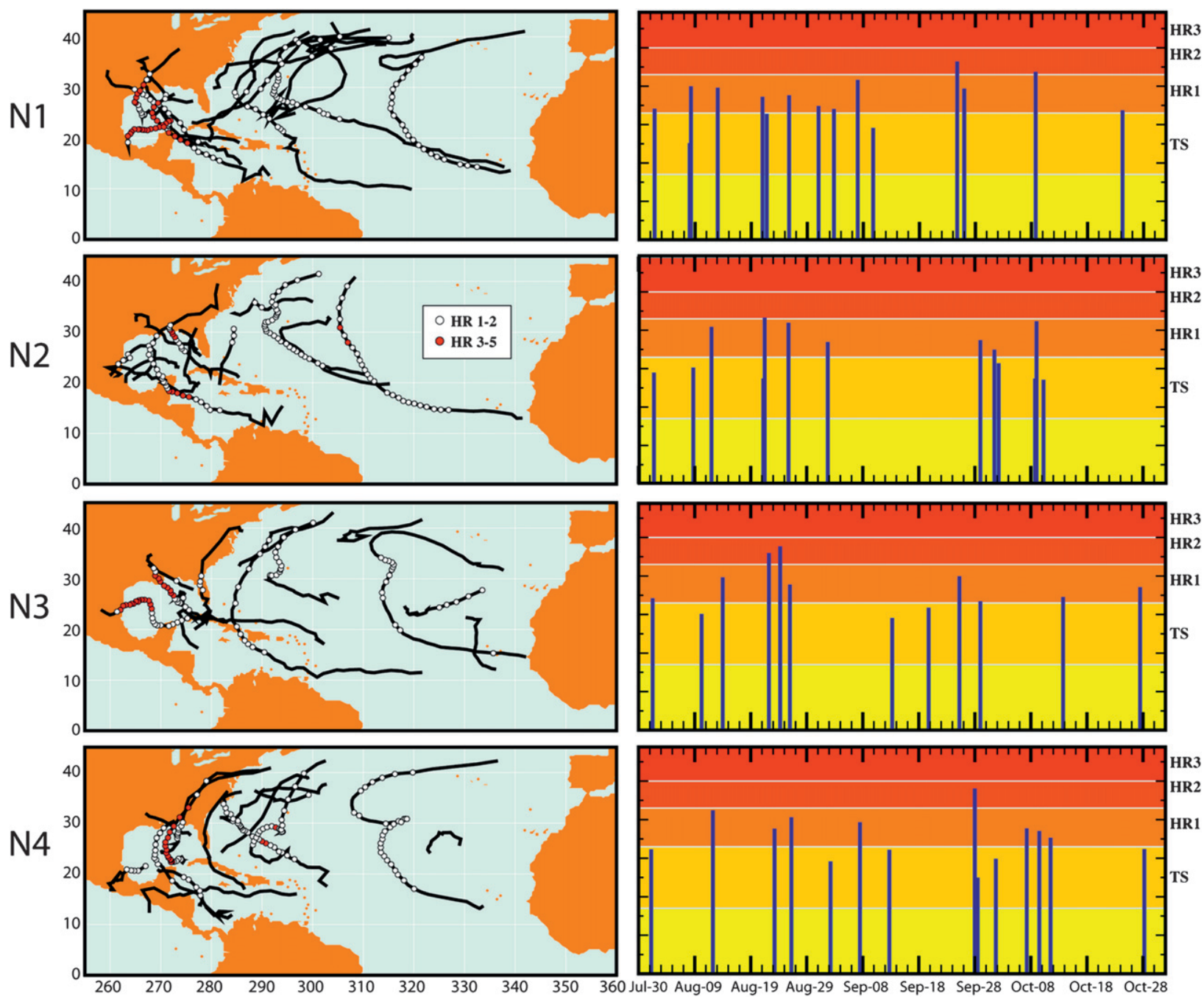

FIG. 2. (left) Storm trajectories and (right) genesis times in each of four realizations of the 1995 season. The colors of the circles along the trajectories correspond to minimum-pressure categories. The heights of the bars indicate maximum intensities based on wind speed, with categories indicated on the vertical axis. The tracks of the observed 1995 storms are shown in Fig. $7 \mathrm{~b}$ of K07.

directly associated with the MDR-averaged thermal stratification and SST by subtracting the results of second kind of perturbation from those of the first kind.

Linearity could be checked via the inverse experiment in which all fields except the MDR-averaged temperatures are perturbed. The importance of horizontal shear could be isolated by adding a nondivergent barotropic circulation to the large-scale flow. We leave these additional experiments for a later time. We emphasize again that the temperature-only perturbation implicitly includes changes in the absolute humidity profile because of the temperature dependence of the saturation specific humidity. This is the biggest source of low-frequency variability in the analyzed moisture field.

We investigate two perturbation scenarios. The first is determined by the linear trend over the period studied by $\mathrm{K} 07$, here extended to 2006 as in K08. Then we examine projected changes due to global warming. We generate ensembles of four realizations per environment to make the seasonal statistics as robust as possible.

\section{The ensembles}

The experiments are initialized from the 0000 UTC reanalysis of 27, 28, 29, or 30 July and integrated through the end of October for a given year. From 1 August onward, the 6-hourly model output is objectively analyzed for occurrences of tropical storms and hurricanes. The storm identification and tracking procedure is detailed in appendix A of K07. The different start times produce different ensemble members. The number of members in the ensembles is limited by computer resources. 

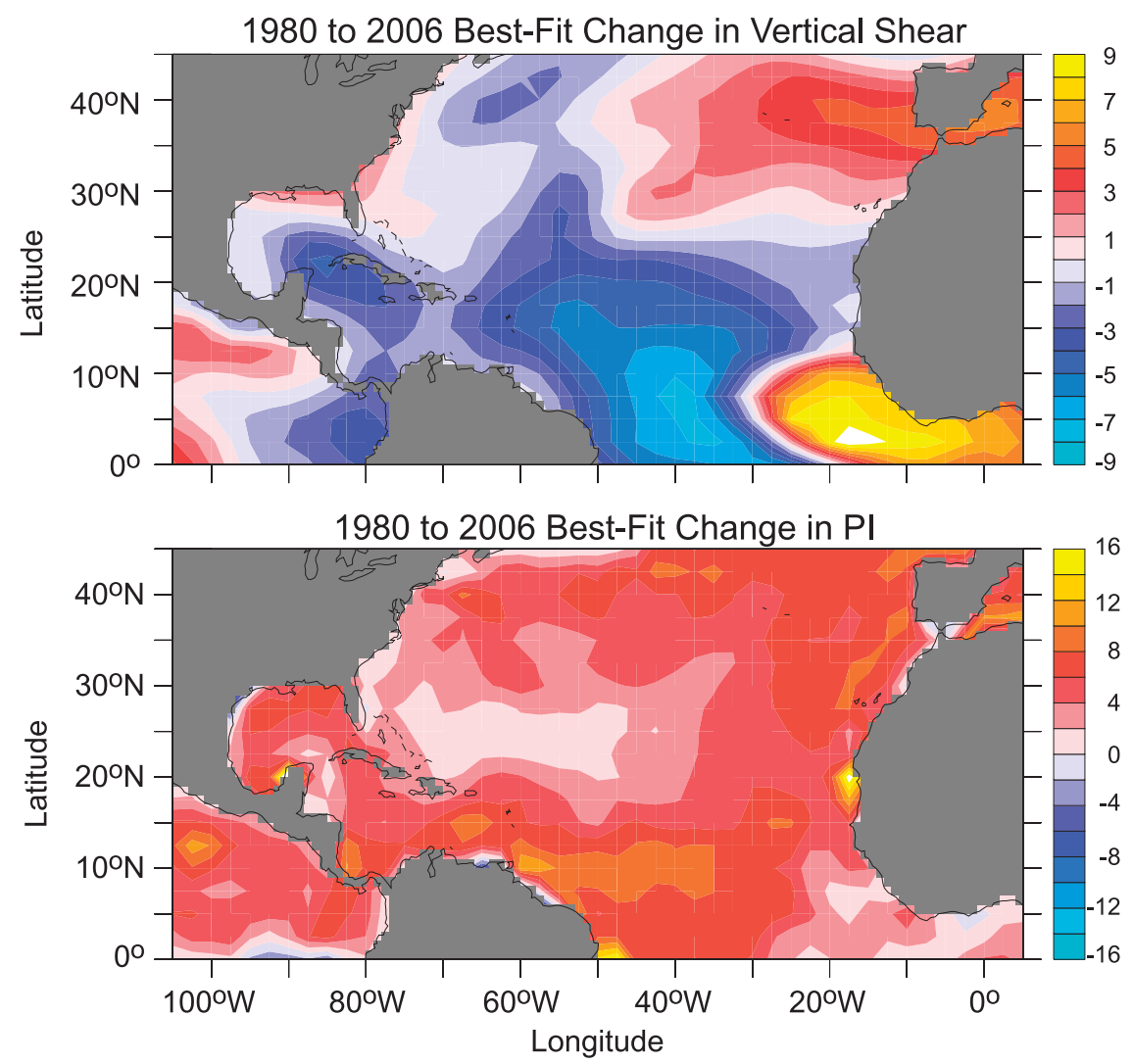

FIG. 3. Changes in (top) vertical shear and (bottom) maximum potential intensity (both $\mathrm{m} \mathrm{s}^{-1}$ ) associated with the linear trends in wind, temperature, and humidity from 1980 to 2006. Data are from NCEP-1 reanalysis. Shear is measured between 850 and $200 \mathrm{hPa}$.

Figure 2 provides an indication of the degree of variability and independence between solutions for the same season obtained using slightly different initial conditions. It shows tropical storm trajectory maps from each of the four realizations of the very active 1995 season. These all use the unperturbed time-dependent NCEP-1 environment and are referred to as the control ensemble. Storm strength is indicated by the colors along the tracks. The diagram beside each map gives the time record of the genesis of the model storms. The height of the bars gives the maximum intensity attained. For this year, the number of model storms ranged from 12 to 15 , while the observed count was 15 .

The substantial differences between the tracks and timings in the different realizations make it clear that neither the boundary forcing nor the interior nudging in the model is strongly constraining the development of individual disturbances. Given this chaotic component to model storm genesis, ensemble averaging is needed to help extract the part of the solution forced by the largescale inputs. At the same time, the magnitude of the chaotic component is itself of interest, since the level of "noise" in the system determines the potential predict- ability of these statistics given perfect large-scale information, including perfect information on the boundaries.

There are exceptional instances in which the imposed forcing or initialization creates coherence across the ensemble. All four solutions for 1995 show a development in the northern Gulf of Mexico on 1 August. This is because the initial fields for this year, taken from the last few days of July, contain a weak representation of Tropical Storm Dean, which amplified to tropical storm strength in all four solutions. Of the 26 seasons simulated by K07, only two others (1980 and 2004) developed a storm in this way from an initial depression.

We found no other cases of genesis events occurring at the same time and location as an observed storm and in each member of the ensemble. However, it is possible for synoptic-scale waves forced at the eastern boundary to synchronize storms off the coast of Africa in different ensemble members. There is one clear instance of this synchronization in the 1995 experiment. In the realizations labeled N2 and N3 in Fig. 1, storm genesis occurred at the same location near the African coast on 25 August (there was no corresponding observed storm). The significance 


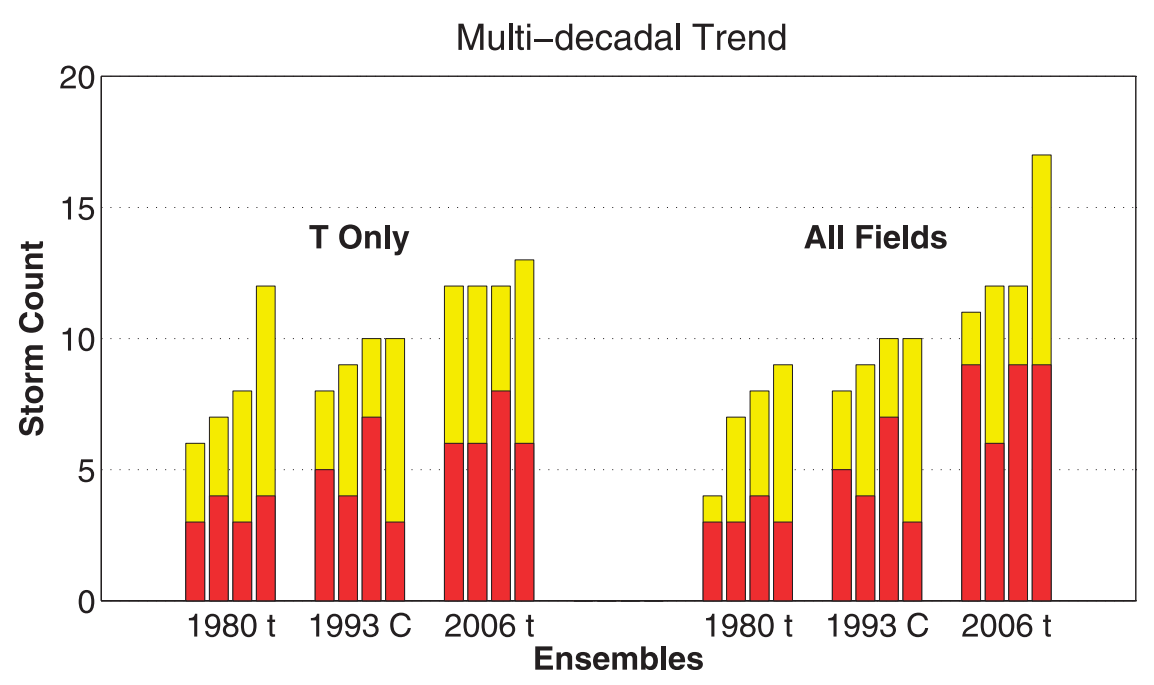

FIG. 4. Number of tropical cyclones (yellow) and hurricanes (red) grouped by ensemble for the start (1980t) and end (2006t) of the 26-yr linear trend in (left) temperature and SST only and (right) all environmental fields. The control ensemble for the weather year, 1993, is also shown between $1980 t$ and $2006 t$ for both types of perturbation.

of the easterly-wave climatology for Atlantic development is an important question that we do not directly address in this study.

For estimating the noise level in the seasonal-mean statistics, we would prefer a larger ensemble than we were able to generate for any individual season. In appendix A, we describe a way to exploit the multiyear results of K07 and K08 to reach a more robust measure of the noise in the system. In our analysis of the ensemble means to follow, we consider $t$ tests based on both the small-ensemble variances and variances derived independently from the multiyear datasets in $\mathrm{K} 07$ and $\mathrm{K} 08$.

\section{The 1980-2006 trend}

For the long-term trend, we use the least squares linear fit to the NCEP reanalysis fields for 1980-2006. The impact on storm statistics is estimated by perturbing the ASO climate of 1993 with plus or minus half the 26-yr linear variation. The subseasonal variations (weather) at the boundaries and in the interior nudging are from 1993. The 1993 reanalysis is close to the 27 -season mean in terms of storms counts, vertical shear, and PI. Subtracting half the trend yields what we term the $1980 t$ season; adding half the trend yields the 2006t season.

The spatial patterns of trends in vertical shear and hurricane PI are shown in Fig. 3. The magnitude of the shear and the potential intensity are computed after applying the trend to the reanalysis fields and timeaveraging the sum and difference over ASO. The figure therefore shows the difference in low-frequency shear and PI between the two environments actually used in the experiments. As in Fig. 1, the shear is defined using the standard levels 200 and $850 \mathrm{hPa}$ and the PI is defined using the method of Bister and Emanuel (2002).

In the all-fields perturbations, we change the seasonalmean temperature, relative humidity, wind, and sea surface temperature in the reanalysis. This full perturbation produces the differences shown in Fig. 3 . In the $T$-only perturbations, we change only the air temperature and sea surface temperature by imposing a single perturbation profile obtained by averaging the temperature trends over the MDR. In this experiment, we hold the wind and relative humidity, as well as the horizontally varying part of the temperature fields, at 1993 values. We generate four realizations for each of the perturbed environments and also generate a four-member control ensemble by combining three new control realizations of 1993 with the Model 2 solution previously obtained by K07.

In all of these experiments, the "weather" in the fields that drive the boundaries and the large scales in the interior is identical. By not changing the higher frequencies in the forcing, we are testing whether the model-generated trend in seasonal statistics reported in K07 can be explained just from the influence of the trend in the seasonal means. An affirmative answer would help justify the method used in $\mathrm{K} 08$ to assess the response to global warming, since only the projected change in seasonalmean climate is used there to perturb the system.

The individual storm counts are shown in Fig. 4. The realizations within each ensemble are reordered according to the total storm count. The 1993 control ensemble, 1993C, is shown between $1980 t$ and $2006 t$ for both perturbations. If the seasonal-mean anomaly is the 
TABLE 1a. Number of tropical storms at beginning and end of linear trend in environmental fields (columns labeled " $1980 t$ " and "2006t") and corresponding net change and percentage change (columns labeled "change" and "percent"). The percentage change is relative to the mean of $1980 t$ and $2006 t$ for each scenario, with bold indicating significance at the $95 \%$ level for the ensembles based on the ensemble variances. Values in row 1 are determined from observations; in row 2 from the Model2 solutions reported in $\mathrm{K} 07$ and $\mathrm{K} 08$; in row 3 from the ensembles with all fields perturbed; and in row 4 from the ensembles with only temperatures perturbed. The average for the 1993 control ensemble is 9.25 tropical storms.

\begin{tabular}{ccccc}
\hline \hline TSs $N(1993)=9.25$ & $1980 t$ & $2006 t$ & Change & Percent \\
\hline Observed & 6.0 & 12.0 & +6 & +67 \\
K07, K08 & 7.8 & 14.5 & +6.7 & +60 \\
All fields & 7.0 & 13.0 & +6.0 & $+\mathbf{6 0}$ \\
$T$ only & 8.25 & 12.25 & +4.0 & $+\mathbf{3 9}$ \\
\hline
\end{tabular}

key aspect of the forcing input into the downscaling model, the contrast between the 1980t and 2006t ensembles for a given statistic should match the trend for the same statistic produced by the full set of 27 seasons simulated by K07 and K08. The ensemble-mean storm counts are listed in Tables 1a and $1 \mathrm{~b}$ for tropical storms and hurricanes, respectively.

For the $27 \mathrm{yr}$ of simulation available by combining $\mathrm{K} 07$ and $\mathrm{K} 08$, the linear trend in tropical storm count is roughly 8 to 14 . The trend in the observed count is 6 to 12 . The model is known to be biased toward too many tropical storms compared to observations (K07). The number of model hurricanes in K07 and K08 increases from about four to eight during the period, compared to the observed increase from three to seven. The size of these trends is well above the noise level inferred from the multiyear variance estimates mentioned in the previous section and in appendix A.

The solutions driven by the trend in the full ASO environment show similar increases. Thus, with all fields perturbed, the ensemble-mean storm counts are 7.0, 9.25, and 13.0 for the seasons $1980 t, 1993$, and 2006t, respectively. Hurricanes total $3.25,4.75$, and 8.25 , respectively. When only the temperature profile and SST are perturbed, the trends are somewhat weaker. In those ensembles, we find $8.25,9.25$, and 12.25 for tropical cyclones, and 3.5, 4.75, and 6.5 for hurricanes. According to a $t$ test of the four-member ensembles, the changes in

TABLE 1b. As in Table 1a, but showing numbers of hurricanes. The 1993 control ensemble had an average of 4.75 hurricanes.

\begin{tabular}{cllcc}
\hline \hline Hurricanes $N(1993)=4.75$ & $1980 t$ & $2006 t$ & Change & Percent \\
\hline Observed & 3.4 & 7.2 & +3.8 & +72 \\
K07, K08 & 4.2 & 8.2 & +4.0 & +65 \\
All fields & 3.25 & 8.25 & +5.0 & $+\mathbf{8 7}$ \\
$T$ only & 3.5 & 6.5 & +3.0 & $+\mathbf{6 0}$ \\
\hline
\end{tabular}

TABLE 1c. As in Table 1a, but showing accumulated cyclone energy (ACE; $10^{4} \mathrm{kn}^{2}$ ). The $1993 \mathrm{control}$ ensemble had an average ACE of $78.2 \times 10^{4} \mathrm{kn}^{2}$.

\begin{tabular}{cccrr}
\hline \hline ACE $A(1993)=78.2$ & $1980 t$ & $2006 t$ & Change & Percent \\
\hline Observed & 47.3 & 143.7 & +96.4 & +101 \\
K07, K08 & 60.2 & 143.2 & +83.0 & +82 \\
All fields & 53.8 & 158.5 & +104.7 & $+\mathbf{9 9}$ \\
$T$ only & 69.9 & 123.7 & +53.8 & $+\mathbf{5 6}$ \\
\hline
\end{tabular}

the number of tropical storms and hurricanes between $1980 t$ and $2006 t$ are significant at the $95 \%$ level, as indicated by bold italics in the last column of Table $1 \mathrm{a}$. This is also true when we use the multiyear variances as described in appendix A. For the hurricane counts, the difference between the all-fields and T-only trends is significant at the $95 \%$ level, but for the total storm counts, the difference is only significant at a much lower level of about $60 \%$.

Storm counts do not measure longevity or intensity. Two familiar measures of cumulative storm activity are the power dissipation index (PDI; Emanuel 2005) and the accumulated cyclone energy (Bell et al. 2006). PDI is a time integral of the cube of the wind speed maximum, which is interpreted as being proportional to the rate of dissipation of kinetic energy. ACE is a time integral of the kinetic energy itself, normalized by $6 \mathrm{~h}$ to retain energy units. Being nonlinear as well as cumulative, these statistics are sensitive to cyclone intensity and longevity as well as to total number.

Tables 1c and 1d list the ACE and PDI for the linear trend experiments. We again use the last column of the tables to show the percentage change relative to the average for the experiment or time series. Relative to the model's 27-yr average, the linear growth of ACE in the $\mathrm{K} 07$ and $\mathrm{K} 08$ simulations is $82 \%$, somewhat less than the observed factor of $101 \%$ (relative to the observed average). In our all-fields experiments, ACE increases by $99 \%$ of the average of $1980 t$ and $2006 t$, whereas the temperature-only experiments show a relative increase of $56 \%$. The linear increase in PDI over the $27 \mathrm{yr}$ simulated by $\mathrm{K} 07$ and $\mathrm{K} 08$ is $91 \%$, an underestimate of the observed $111 \%$ increase. The all-fields ensembles increase the PDI by $106 \%$, whereas the temperature-only

TABLE 1d. As in Table 1a, but showing the power dissipation index (PDI; $10^{9} \mathrm{~m}^{3} \mathrm{~s}^{-2}$ ). The 1993 control ensemble had an average PDI of $137.0 \times 10^{9} \mathrm{~m}^{3} \mathrm{~s}^{-2}$.

\begin{tabular}{crrrr}
\hline \hline PDI $P(1993)=137.0$ & $1980 t$ & $2006 t$ & Change & Percent \\
\hline Observed & 105.2 & 365.2 & +260.1 & +111 \\
K07, K08 & 99.2 & 263.6 & +164.4 & +91 \\
All fields & 89.1 & 291.9 & +202.8 & $+\mathbf{1 0 6}$ \\
$T$ only & 119.9 & 230.3 & +110.4 & $+\mathbf{6 3}$ \\
\hline
\end{tabular}


ensembles increase it by $63 \%$, relative to the average of $1980 t$ and $2006 t$.

The increases in ACE and PDI are significant at the 95\% level for both the all-fields and $T$-only experiments. For both statistics, the differences between the all-fields and $T$-only increases are significant at the $90 \%$ level. Using the multi-year variance of PDI (see appendix A) raises the significance level of the difference between all-fields and $T$-only to $95 \%$ for that statistic.

In summary, the trend in seasonal-mean fields reproduces the full trend in the $27 \mathrm{yr}$ of modeled (and observed) storm activity quite well, despite somewhat overshooting the relative increase in the case of hurricanes. The thermal part of the environmental trend (MDR temperature profile and SST with fixed relative humidity) provides more than half the increase in storm counts as well as in ACE and PDI.

\section{A climate change scenario}

K08 used a method similar to the present one to assess the impact of projected forced climate change on Atlantic tropical storm activity in the late twenty-first century. They perturbed the seasonal-mean environment of the same regional model as used here with a multiyear, multimodel, August-to-October average change in wind, temperature, sea surface temperature, and humidity. The perturbation was an 18-model average of differences between 2081-2100 and 2001-2020 based on the Intergovernmental Panel on Climate Change (IPCC) A1B scenario.

The main result in K08 was a significant mean reduction in storm activity, including a $27 \%$ decrease in tropical storm frequency and an $18 \%$ decrease in hurricane frequency. Because of some distortion of the wind-pressure relationship for strong storms in the model, as described in K07, "major" hurricane counts depend on whether one uses standard wind or pressure criteria. The model produces category- 3 and -4 hurricanes according to the minimum surface pressure criterion, but not according to the maximum wind criterion. The number of such major hurricanes defined according to the pressure criterion diminished only minimally (down $8 \%$ ) in the model's 21st-century projection.

To further investigate the causes of the overall storm suppression in the model, we consider perturbations to the active year 1995. For the control case, we add three new realizations to the present-climate solution obtained by K07. Then we augment the existing K08 solution with three new warm-climate realizations of 1995 in which the seasonal mean is shifted by all of the climate change fields. Finally, we generate a four-member ensemble in which only the sea surface and atmospheric tempera- tures are shifted by the multimodel climate change projection, averaged over the MDR to create a single thermodynamic change profile.

The climate change fields used here and in K08 have been examined in detail by Vecchi and Soden (2007a). Most notably, the vertical shear in the Caribbean Sea increases by around $10 \%$ per degree of global warming. The sign of this feature is quite robust across the models used in the average. The average change in PI in the tropical Atlantic is relatively small. The sign of the change in the center of the basin varies between models, but there is a robust contrast between increased PI in the west and decreased PI in the east (G. Vecchi 2009, personal communication). Similar patterns in shear and PI appear when we perturb 1995 with the climate change fields and reevaluate the indices. The changes are shown in Fig. 5. The MDR-averaged increase in shear is around $10 \%$, and this jumps to $15 \%$ in the western half of the MDR. The increase in PI averaged across the MDR is around 2\%.

The storm counts for the individual experiments are shown in Fig. 6. Ensemble-mean statistics are given in Tables 2a-d. For the all-fields ensembles, the mean reduction in total storms is $22 \%$, whereas the hurricanes are reduced by $32 \%$. The difference between the sizes of these reductions is not statistically significant. For an indication of how storm suppression depends on storm strength, we have more confidence in the multiyear average reductions obtained by $\mathrm{K} 08$ for the same kind of all-fields perturbation ( $27 \%$ and $18 \%$, respectively). The ACE and PDI both decrease by $27 \%$. The impact of climate change in the temperature-only experiments, where the SST and atmospheric temperature profile is altered to resemble the future MDR, is negligible.

In the all-fields global warming ensemble, the average decreases in total storm count, PDI, and ACE are all significant at the $90 \%$ level when the $t$ test is performed with the ensemble variances. According to this test, only the decrease in the hurricane count is significant at the $95 \%$ level. When the multiyear variance estimates (appendix A) are used in the $t$ test, all of the decreases due to the all-fields perturbation are significant at the $95 \%$ level. The various small changes resulting from the $T$-only perturbation are not significant.

\section{Discussion and conclusions}

A hurricane-resolving dynamical model nested in global reanalysis fields has been used to estimate the relative importance of the thermodynamic profile and other environmental factors, including vertical shear, in explaining tropical storm variability. Two types of variability - the recent multidecadal trend and a late 21st-century global-warming scenario-are explored. In 

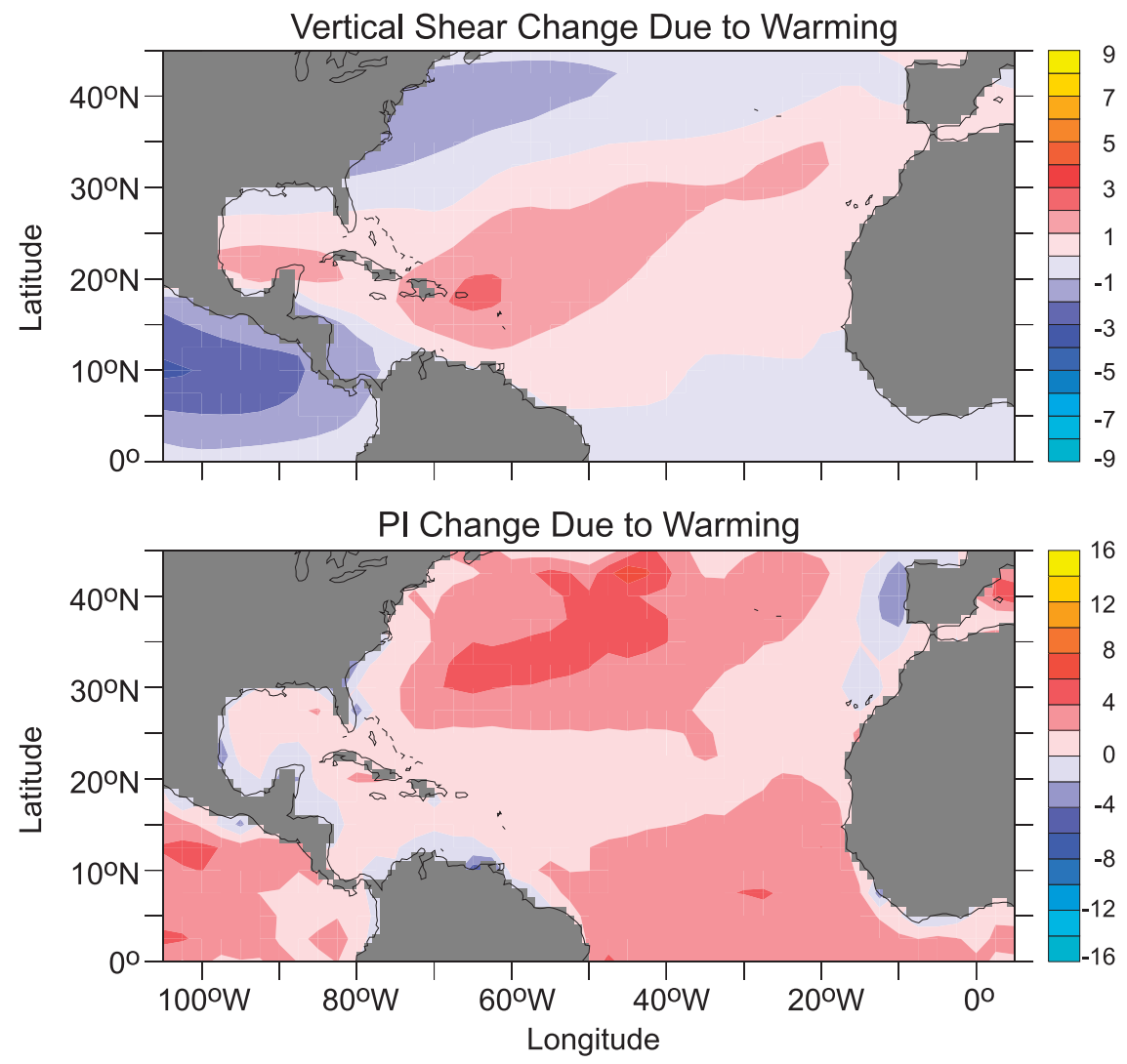

FIG. 5. Changes due to the global warming perturbation in (top) vertical shear and (bottom) hurricane potential intensity (both $\mathrm{m} \mathrm{s}^{-1}$ ) for the year 1995. The perturbation is a Coupled Model Intercomparison Project (third phase; CMIP3) multimodel average change for the late 21st century assuming the A1B forcing scenario. Fields are defined as in Fig. 3.

the first case, season-by-season simulations capture the observed differences in storm counts fairly well by roughly doubling the number between 1980 and 2006. In the global-warming scenario, the model projects a decrease in Atlantic tropical storm and hurricane counts.

In the historical situation, we find that the seasonalmean fields are sufficient to reproduce the long-term trends in the model statistics, which are similar to the observations. When we limit the perturbation of the ASO environment to the horizontally averaged temperature profile (with fixed relative humidity) and SST, we recover more than half of the upward trend in the storm counts and cumulative statistics (ACE and PDI).

Capturing most of the long-term trends in storm statistics with these experiments suggests that whatever trends may exist in the "weather" (subseasonal variability) are of secondary importance. We have more evidence in support of this conclusion (that the seasonal mean is the dominant influence), based on experimentation with the years 1982 and 1995. Those results are presented in appendix B. The overriding importance of the seasonal-mean environment in the model's multi- decadal trend helps to justify the approach used in the climate change study by $\mathrm{K} 08$, in which forced changes at subseasonal frequencies were neglected.

Our results for the climate change scenario differ from those of the multidecadal trend in that the thermal perturbation within the projected climate change by itself had little effect on model storm statistics. We therefore

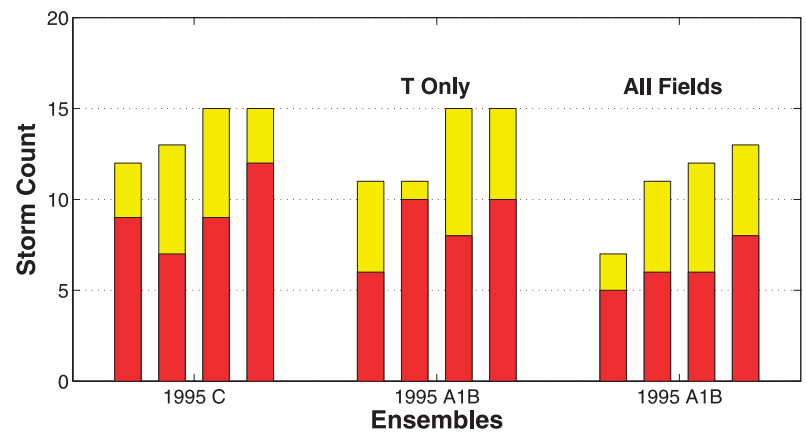

FIG. 6. Number of tropical cyclones (yellow) and hurricanes (red) grouped by ensemble for the 1995 control and the 1995 temperature-only and all-fields climate change perturbations. 
TABLE 2a. Number of tropical storms in the 1995 control ensemble and in the ensembles obtained by perturbing the 1995 seasonal mean with the complete climate change fields or only the temperature profile and SST from the climate change fields.

\begin{tabular}{lcccc}
\hline \hline \multicolumn{1}{c}{ TSs } & 1995 & A1B & Change & Percent \\
\hline Control & 13.75 & & & \\
All fields & & 10.75 & -3.00 & -22 \\
$T$ only & & 13.00 & -0.75 & -5 \\
\hline
\end{tabular}

speculate, given its known importance for tropical storm development, that vertical shear is largely responsible for the storm suppression. (While the experiments here do not test whether other changes-relative humidity, relative vorticity, and large-scale subsidence, to name a few-are important, these changes are so small in the climate change projections that we think it unlikely.)

Global models running with greenhouse-gas forcing do not substantially increase hurricane PI or other measures of moist stability in the 21st century. Accordingly, it is not surprising that the SST and temperature stratification part of the climate change fields explains little if any of the storm suppression simulated by K08. Increases in the vertical moisture gradient, a possible mechanism for storm suppression (Emanuel 2008), are included implicitly in the temperature-only experiments.

In our model, any effect of the mean thermal state and SST on the multidecadal trend is not due to the increase in local Atlantic SST alone, since a substantial SST increase occurs in both the 1980-2006 trend and the climate change scenario, whereas only the 1980-2006 trend yielded increases in model storminess. The relevant characteristic of the thermal state is presumably the hurricane potential intensity. If the trend toward increasing PI in the NCEP reanalysis since 1980 is unrealistic, then the model results here and in $\mathrm{K} 07$ are likely overemphasizing the trend in storm and hurricane counts. It would follow that the model may be matching some of the trend in the observed counts for the wrong reason.

The sensitivity of the model's tropical storm activity to projected changes in vertical shear (or other aspects of the circulation) is evidently substantial. The statisticaldynamical genesis parameter proposed by Emanuel (2008), for example, is of the form

TABLE 2b. As in Table 2a, but for the number of hurricanes. The statistically significant changes are indicated in the last column by boldface.

\begin{tabular}{lcccr}
\hline \hline Hurricanes & 1995 & A1B & Change & Percent \\
\hline Control & 9.25 & & & \\
All fields & & 6.25 & -3.00 & $-\mathbf{3 2}$ \\
$T$ only & & 8.50 & -0.75 & -8 \\
\hline
\end{tabular}

TABLE 2c. As in Table 2a, but for the accumulated cyclone energy.

\begin{tabular}{lcrrr}
\hline \hline ACE & 1995 & A1B & Change & Percent \\
\hline Control & 116.9 & & & \\
All fields & & 85.3 & -31.6 & -27 \\
$T$ only & & 118.2 & +1.3 & +1 \\
\hline
\end{tabular}

$$
\mathrm{GPI} \propto\left(V_{\text {pot }}\right)^{3}\left(1+0.1 V_{\text {shear }}\right)^{-2},
$$

where $V_{\text {pot }}$ refers to the hurricane PI and $V_{\text {shear }}$ to the vertical shear. The area averaged $2 \%$ increase in PI corresponds to a $6 \%$ increase in GPI, which is too small to be discernible. The $10 \%$ increase in shear (over its background value of $14 \mathrm{~m} \mathrm{~s}^{-1}$ ) induces a decrease in GPI of $9 \%$, which is smaller than the reduction in model storm counts seen in $\mathrm{K} 08$ and in the all-fields simulations here. However, in the western half of the MDR, the $15 \%$ increase in shear produces a $15 \%$ decrease in GPI, which is closer to the simulated reduction across the full basin. Contributions to the genesis index from relative humidity and low-level circulation anomalies due to climate change are much smaller than contributions from PI and vertical shear (Vecchi and Soden 2007a).

The present model, using objective storm counting, is biased toward genesis in the western (at the expense of the eastern) part of the basin (K07). For example, the number of August-October genesis events east of $50^{\circ}$ averages 3.0 in the 1995 control, compared to 7 in the observations. We believe this is largely a result of the systematic bias in intensity rather than in the preferred location of organization. Otherwise, there may be some concern that model storms are excessively vulnerable to unfavorable conditions in the Caribbean Sea.

We summarize our most important findings as follows: For the multidecadal trend, changes in the seasonalmean thermodynamic environment account for more than half of the observed increase in hurricane and tropical cyclone numbers and in the cumulative statistics, ACE and PDI. For the 21st-century climate change scenario, the model's projected reduction in Atlantic tropical cyclone activity appears driven mostly by circulation changes, notably the increased seasonal-mean vertical shear in the western Atlantic and Caribbean.

TABLE 2d. As in Table 2a, but for the power dissipation index.

\begin{tabular}{lcccr}
\hline \hline \multicolumn{1}{c}{ PDI } & 1995 & A1B & Change & Percent \\
\hline Control & 214.5 & & & \\
All fields & & 157.3 & -57.2 & -27 \\
$T$ only & & 219.3 & +4.8 & +2 \\
\hline
\end{tabular}


Acknowledgments. We would like to acknowledge helpful analysis and suggestions by Bob Tuleya, Gabe Vecchi, and Ming Zhao. Chris Kerr helped with the numerical modeling.

\section{APPENDIX A}

\section{Statistical Significance of the Model Sensitivity}

For significance testing, we have relied on a simple $t$ test, based on an assumption that the results are sampled from normal distributions. But is it safe to assume that the possible outcomes are normally distributed?

The number of tropical storms or hurricanes in a season is a count of genesis events based on an arbitrary threshold for storm intensity. If we approximate the dynamical system with a stochastic model in which genesis per unit time occurs at some probability fixed by the large-scale environment, storm frequency statistics would follow the corresponding binomial probability distribution. One could use this distribution to test for significance were it known how to subdivide time and space into the equivalent of independent trials (the distribution is approximated by a Poisson distribution if the probability of genesis within the trials is small).

Fortunately, a binomial distribution is nearly normal (the mean value is well separated from zero in standard deviation units) if the probability is much greater than $1 /(N+1)$, where $N$ is the number of trials. As this is practically the same as having a large storm count, one does not need to determine a trial number, $N$ (which merely has to be large enough to overcome skewness in the distribution). We conclude that it is reasonable to consider the possible outcomes for storm counts in our experiments to be normally distributed. This would not be the case for subbasins small enough that the mean number of events approached unity.

Significance testing is still compromised by small sample size. A close approximation to a much larger ensemble than we were able to generate for individual years is available by combining the multiyear results of K07 and K08. The only difference between the 26 sets of seasonal statistics (1980 to 2005) generated in each of these studies is due to the global warming perturbation added to the reanalysis in the later study. To use the combined results for noise estimation, we propose that same-year differences can be viewed as the climate change signal plus pure noise, with both the mean climate change and noise being independent of year. These differences are plotted as histograms in Fig. 3 of K08. The standard deviation is 2.4 for both total storms and hurricanes. We suggest this is a reasonable measure of the ensemble spread when differences are taken between two years. Dividing the variance by 2 , we estimate that the standard deviation for an individual year is 1.7 storms. We cannot say how much of the variance is natural and how much is due to unphysical aspects of the model.

Within the much smaller sample provided by our 1995 control ensemble, the tropical storm counts have a standard deviation of 1.3 and the hurricane counts have a standard deviation of 1.8. These are not far from the values obtained with the larger sample. For the cumulative statistics, the standard deviation from the multiyear dataset is $23.0 \times 10^{4} \mathrm{kn}^{2}$ for the ACE and $45.5 \times 10^{9}$ $\mathrm{m}^{3} \mathrm{~s}^{-2}$ for the PDI. These are also similar to the 1995 control ensemble, which gives $22.0 \times 10^{4} \mathrm{kn}^{2}$ and $40.8 \times$ $10^{9} \mathrm{~m}^{3} \mathrm{~s}^{-2}$, respectively.

In addition to the $t$ test based on the small-ensemble variances, we consider an alternative $t$ test based on the multimodel variances using the statistic

$$
\varphi=\frac{\left(\bar{u}_{1}-\bar{u}_{2}\right)}{\sqrt{2 \sigma^{2} / N}},
$$

where $\bar{u}_{1}$ and $\bar{u}_{2}$ are the sample means being contrasted, $\sigma^{2}$ is the variance of the larger sample, and $N$ remains the size of the small ensemble. This statistic is associated with the $t$ distribution defined by the size of the larger sample, which has 25 degrees of freedom.

\section{APPENDIX B}

\section{An Interannual Contrast}

Between isolated years, the pattern of change in the environment may be quite different from long-term trends. For an indication of the importance of the seasonal mean compared to subseasonal frequencies in driving interannual tropical storm variability, we created a mixed-environment ensemble in which the subseasonal variability of 1995 is replaced by that of the much less active year 1982. Equivalently, we set up an experiment for 1982 in which the ASO averages were replaced by those of 1995. A control ensemble for 1995 was mentioned in section 3 . We also produced a control

TABLE B1a. Number of observed and simulated tropical storms in 1982 and 1995. Values in row 1 are from observations; in row 2 from the control ensembles; and in row 3 from the experiments with mixed environments where the year providing the seasonal mean is specified by the column header. The case marked with an asterisk is a single experiment; otherwise, the model results are four-member ensemble averages.

\begin{tabular}{lll}
\hline \multicolumn{1}{c}{ TSs } & 1982 & 1995 \\
\hline Observed & 4 & 15 \\
Control & 6.75 & 13.75 \\
Mixed & $6^{*}$ & 16.25 \\
\hline
\end{tabular}


TABLE B1b. As in Table B1a, but showing numbers of hurricanes.

\begin{tabular}{llc}
\hline \hline Hurricanes & 1982 & 1995 \\
\hline Observed & 1 & 10 \\
Control & 3.50 & 9.25 \\
Mixed & $3^{*}$ & 8.75 \\
\hline
\end{tabular}

ensemble for 1982. The resulting mean storm counts are shown in Tables B1a and B1b for tropical storms and hurricanes, respectively.

The mixed-environment ensemble with 1995 seasonalmean fields (labeled "mixed" and "1995" in the tables) closely resembles the 1995 control, although it exaggerates the increase in total storms over 1982. A single realization with the reversed mixed environment (1982 mean plus 1995 higher frequencies; marked with an asterisk in the tables) produces a good match to the 1982 control ensemble. At least for these years, changing the 3-month mean environment is sufficient to reproduce the differences in storm counts obtained using the full variation of conditions between the years.

\section{REFERENCES}

Bell, G. D., E. Blake, K. C. Mo, C. W. Landsea, R. Pasch, M. Chelliah, S. B. Goldenberg, and H. J. Diamond, 2006: The record-breaking 2005 Atlantic hurricane season. Bull. Amer. Meteor. Soc., 87, 544-545.

Bister, M., and K. A. Emanuel, 2002: Low frequency variability of tropical cyclone potential intensity. 1. Interannual to interdecadal variability. J. Geophys. Res., 107, 4801, doi:10.1029/ 2001JD000776.

Carmargo, S. J., K. A. Emanuel, and A. H. Sobel, 2007: Use of genesis potential index to diagnose ENSO effects upon tropical cyclone genesis. J. Climate, 20, 4819-4834.

DeMaria, M., 1996: The effect of vertical shear on tropical cyclone intensity change. J. Atmos. Sci., 53, 2076-2087.

_ J. A. Knaff, and B. H. Connell, 2001: A tropical cyclone genesis parameter for the tropical Atlantic. Wea. Forecasting, 16, 219-233.

Emanuel, K. A., 1986: The maximum potential intensity of hurricanes. J. Atmos. Sci., 45, 1143-1155.

_ 2005: Increasing destructiveness of tropical cyclones over the past 30 years. Nature, 436, 686-688.

_ 2007: Environmental factors affecting tropical cyclone power dissipation. J. Climate, 20, 5497-5509.

- 2008: The hurricane-climate connection. Bull. Amer. Meteor. Soc., 89, ES10-ES20.

Goldenberg, S. B., and L. J. Shapiro, 1996: Physical mechanisms for the association of El Niño and West African rainfall with Atlantic major hurricane activity. J. Climate, 9, 1169-1187.

_ C. W. Landsea, A. M. Mesta-Nunez, and W. M. Gray, 2001: The recent increase in Atlantic hurricane activity: Causes and implications. Science, 293, 474-479.

Gray, W. M., 1968: Global view of the origin of tropical disturbances and storms. Mon. Wea. Rev., 96, 669-700.

, C. W. Landsea, P. W. Pielke Jr., and K. J. Berry, 1994: Predicting Atlantic Basin seasonal tropical cyclone activity by 1 June. Wea. Forecasting, 9, 103-115.
Hebert, P. J., 1978: Intensification criteria for tropical depressions of the western North Atlantic. Mon. Wea. Rev., 106, 831-840.

Holland, G., 1997: The maximum potential intensity of tropical cyclones. J. Atmos. Sci., 54, 2519-2541.

Hoyos, C. D., P. A. Agudelo, P. J. Webster, and J. A. Curry, 2006: Deconvolution of the factors contributing to the increase in global hurricane intensity. Science, 312, 94-97.

Kalnay, E., and Coauthors, 1996: The NCEP/NCAR 40-Year Reanalysis Project. Bull. Amer. Meteor. Soc., 77, 437-471.

Karl, T. R., S. J. Hassol, C. D. Miller, and W. L. Murray, cited 2006 : Temperature trends in the lower atmosphere: Steps for understanding and reconciling differences. U.S. Climate Change Science Program, Synthesis and Assessment Product 1.1. [Available online at http://www.climatescience.gov/Library/ sap/sap1-1/finalreport/sap1-1-final-all.pdf.]

Knutson, T. R., J. J. Sirutis, S. T. Garner, I. M. Held, and R. E. Tuleya, 2007: Simulation of the recent multi-decadal increase of Atlantic hurricane activity using a $18-\mathrm{km}$ grid regional model. Bull. Amer. Meteor. Soc., 88, 1549-1565.

,,,--- G. A. Vecchi, and I. M. Held, 2008: Simulated reduction in Atlantic hurricane frequency under twenty-firstcentury warming conditions. Nature Geosci., 1, 359-364, doi:10.1038/ngeo202.

Merrill, R. T., 1988: Environmental influences on hurricane intensification. J. Atmos. Sci., 45, 1678-1687.

Milly, P. C. D., and A. B. Shmakin, 2002: Global modeling of land water and energy balances. Part I: The Land Dynamics (LaD) model. J. Hydrometeor., 3, 283-299.

Nolan, D. S., E. D. Rappin, and K. A. Emanuel, 2007: Tropical cyclogenesis sensitivity to environmental parameters in radiativeconvective equilibrium. Quart. J. Roy. Meteor. Soc., 133, 2085-2107.

Pauluis, O., and S. T. Garner, 2006: Sensitivity of radiativeconvective equilibrium simulations to horizontal resolution. J. Atmos. Sci., 63, 1910-1923.

Santer, B. D., and Coauthors, 2005: Amplification of surface temperature trends and variability in the tropical atmosphere. Science, 309, 1551-1556.

Saunders, M. A., and A. S. Lea, 2008: Large contribution of sea surface warming to recent increase in Atlantic hurricane activity. Nature, 451, 557-560.

Shen, W., R. E. Tuleya, and I. Ginis, 2000: A sensitivity study of the thermodynamic environment on GFDL model hurricane intensity: Implications for global warming. J. Climate, 13, 109-121.

Swanson, K. L., 2008: Nonlocality of Atlantic tropical cyclone intensities. Geochem. Geophys. Geosyst., 9, Q04V01, doi:10.1029/ $2007 \mathrm{GC} 001844$.

Tang, B. H., and J. D. Neelin, 2004: ENSO influence on Atlantic hurricanes via tropospheric warming. J. Geophys. Res., 31, L24204, doi:10.1029/2004GL021072.

Vecchi, G. A., and B. J. Soden, 2007a: Increased tropical Atlantic wind shear in model projections of global warming. Geophys. Res. Lett., 34, L08702, doi:10.1029/2006GL028905.

$\longrightarrow$, and — 2007b: Effect of remote sea surface temperature change on tropical cyclone potential intensity. Nature, 450, 1066-1070.

Vitart, F., and J. L. Anderson, 2001: Sensitivity of Atlantic tropical storm frequency to ENSO and interdecadal variability of SSTs in an ensemble of AGCM integrations. J. Climate, 14, 533-545.

— , and Coauthors, 2007: Dynamically based seasonal forecasts of Atlantic tropical storm activity issued in June by EUROSIP. Geophys. Res. Lett., 34, L16815, doi:10.1029/ 2007GL030740. 